\title{
Efeitos da ingestão prévia de carboidrato de alto índice glicêmico sobre a resposta glicêmica e desempenho durante um treino de força
}

\author{
Ana Paula Trussardi Fayh1,2, Daniel Umpierre1, Katiuce Borges Sapata1,
}

Fernando Matos Dourado Neto ${ }^{1}$ e Alvaro Reischak de Oliveira ${ }^{1}$

\section{RESUMO}

O objetivo deste estudo foi examinar os efeitos da ingestão prévia de carboidrato no desempenho físico e comportamento glicêmico durante o treino de força. Oito voluntários realizaram 2 sessões de exercício de força (7 exercícios com 3 séries na intensidade de $70 \%$ de 1 repetição máxima), nas quais ingeriram bebida composta de carboidrato (maltodextrina) ou placebo. A bebida foi ingerida 15 minutos antes do início da sessão, a ordem das sessões foi randomizada, e essas foram separadas por 7 dias de intervalo. A glicemia foi mensurada em 4 momentos: antes da ingestão da bebida, 15 minutos após a ingestão da bebida, na metade do treino, e ao final do mesmo. O desempenho físico nos dois dias de treino foi influenciado somente pela variação no número das repetições executadas, as quais foram inseridas no cálculo da tonelagem total de treino executada nas respectivas sessões (repetições · séries · carga). A freqüência cardíaca foi continuamente monitorada e a concentração de lactato foi mensurada ao término da sessão. A glicemia esteve aumentada somente aos 15 minutos após a ingestão da bebida com carboidrato (de 98,25 $\pm 17,77 \mathrm{mg} / \mathrm{dL}$ para 133,12 $\pm 22,76 \mathrm{mg} / \mathrm{dL}, \mathrm{p}=0,015$ ), enquanto que no dia da bebida placebo não foram observadas alterações significativas nestes momentos (de 98,25 \pm 13,69mg/ $\mathrm{dL}$ para $94,38 \pm 12,21 \mathrm{mg} / \mathrm{dL}, \mathrm{p}=1,000)$. A tonelagem total de treino, freqüência cardíaca e concentração final de lactato foram semelhantes nos dois treinos de força. Mesmo com o aumento da glicemia pré-exercício após a ingestão da bebida com carboidrato, os resultados do estudo não indicam que a ingestão prévia de carboidrato à sessão de exercício de força pode ser uma suplementação eficaz para aumentar o desempenho físico.

\section{ABSTRACT \\ Effects of pre-exercise ingestion of high glycemic level carbohydrate over the glycemic response and performance during strength training}

The aim of this study was to examine the effects of pre-exercise carbohydrate ingestion on performance and glycemic response during a strength training session. Eight male volunteers performed 2 strength exercise sessions with the ingestion of a carbohydrate (maltodextrin) or placebo drink 15 minutes before each session (7 exercises with 3 sets at $70 \%$ of 1 maximum repetition). The trials

1. Laboratório de Pesquisa do Exercício, Escola de Educação Física, Universidade Federal do Rio Grande do Sul, Porto Alegre, RS.

2. Faculdade de Ciências da Saúde, Centro Universitário Metodista IPA, Porto Alegre, RS.

Aceito em 13/6/07.

Endereço para correspondência: Ana Paula Trussardi Fayh, Rua Felizardo, 750, Jardim Botânico - 90690-200 - Porto Alegre, RS, Brasil. Tels.: (51) 3316-5861/9342-8485, fax: (51) 3316-5811.

E-mail: apfayh@yahoo.com.br
Palavras-chave: Maltodextrina. Glicemia. Exercício.

Keywords: Maltodextrin. Glycemia. Exercise.

were performed 7 days apart from each other and their order was randomized. Glycemia was measured at 4 times: before the drink ingestion, 15 minutes after the drink ingestion, halfway through the training and at the end of the exercise session. The total performance was affected by variation on achieved repetitions in the different days, which were inserted in the total load rate analysis performed in the respective sessions (repetitions . sets . load). Heart rate was continuously monitored and lactate concentration was measured at the end of session. Glycemia increased only at 15 minutes after the carbohydrate drink ingestion (from $98.25 \pm$ $17.77 \mathrm{mg} / \mathrm{dL}$ to $133.12 \pm 22.76 \mathrm{mg} / \mathrm{dL}, p=0.015)$, while on the placebo drink day no significant changes were observed (from 98.25 $\pm 13.69 \mathrm{mg} / \mathrm{dL}$ to $94.38 \pm 12.21 \mathrm{mg} / \mathrm{dL}, p=1.000$ ). The total load rate, heart rate and final lactate concentration were not different in the two strength exercise sessions. Although pre-exercise glycemia was increased after the carbohydrate drink ingestion, the results do not indicate that carbohydrate ingestion before strength exercise session can be an efficient supplementation in order to improve physical performance.

\section{INTRODUÇÃO}

O treinamento de força tornou-se parte integral de um programa de condicionamento físico para atletas e praticantes de exercícios. Com isso, alguns cuidados ergogênicos e estratégias nutricionais são testados no intuito de melhorar o desempenho e aumentar a massa muscular. Partindo do pressuposto que a capacidade de realizar atividade muscular pode ser aumentada pelo conteúdo de glicogênio muscular e hepático, estudos relacionando o consumo de carboidratos, quantidades oferecidas e período de consumo tem sido realizados com o intuito de otimizar o desempenho ${ }^{(1-4)}$. No entanto, em contraste com os efeitos benéficos da ingestão de carboidratos durante o exercício prolongado, a eficácia da ingestão pré-exercício permanece obscura.

Tradicionalmente, a suplementação com carboidratos era associada com o desempenho em exercícios aeróbios. Neste contexto, a suplementação poderia aumentar o tempo de exercício realizado pela elevação da glicose sanguínea e redução do uso de glicogênio muscular ${ }^{(5-6)}$. Entretanto, estudos têm demonstrado que exercícios intermitentes igualmente podem reduzir os estoques de glicogênio muscular(7-8), bem como uma diminuição significativa do glicogênio muscular afeta o trabalho de força quando o estoque inicial de glicogênio é reduzido devido a uma manipulação dietética inadequada ${ }^{(9)}$. O glicogênio pode ser considerado um fator limitante para o desempenho de força, podendo afetar o desempenho pela modificação da funcionalidade do sistema nervoso central através das alterações sanguíneas de glicose (8,10-11). $^{2}$. 
A alimentação pré-treino pode ser considerada um importante recurso ergogênico e fator otimizante de resultados ${ }^{(7,12)}$. No entanto há divergências na literatura referentes à melhora ou não do desempenho quando o exercício é precedido do consumo de carboidratos. Para alguns autores, o consumo de carboidratos préexercício tem sido sugerido como uma alternativa para minimizar a depleção de glicogênio que ocorre ao longo do exercício tanto de força ${ }^{(10,13-14)}$ quanto de resistência aeróbica ${ }^{(2,12)}$. O efeito ergogênico da suplementação de carboidratos parece ocorrer em resposta à elevação dos níveis de glicose que estão associados com a suplementação. Entretanto, poucos estudos se preocuparam em investigar os resultados da ingestão de carboidratos antes do exercício de força sobre o desempenho físico. Haff et al.(4) estudaram os efeitos da suplementação de carboidratos e placebo na habilidade de realizar múltiplas séries de exercício de agachamento durante a segunda sessão de treinamento num único dia. Comparando com o placebo, a suplementação com carboidratos melhorou significativamente o número de séries, repetições e duração do exercício. Porém, o aumento de desempenho não foi constatado em exercícios isocinéticos realizados após uma ingestão de carboidratos antes da sessão de treinamento.

A partir da pequena quantidade de estudos que investigaram a influência da ingestão de carboidratos antes ou durante o exercício de força, nosso objetivo neste estudo foi caracterizar a resposta glicêmica e de desempenho físico numa sessão de exercício de força realizada após ingestão prévia de carboidrato.

\section{MÉTODOS}

\section{Amostra}

Oito indivíduos saudáveis do sexo masculino, com média de idade de 23,9 $\pm 1,5$ anos, foram convidados a participar do estudo mediante exposição do projeto na academia em que treinavam. Como critérios de seleção, os indivíduos deveriam realizar treinamento de força há no mínimo dois anos e não ter ficado sem treinar por mais de 45 dias consecutivos durante este período. O protocolo foi aprovado pelo Comitê de Ética em Pesquisa da Rede Metodista de Educação IPA (no 1100 de 6/8/2004), e os procedimentos foram conduzidos segundo a resolução específica do Conselho Nacional de Saúde (n 196/96). Todos os indivíduos foram informados detalhadamente sobre os procedimentos utilizados e concordaram em participar de maneira voluntária do estudo, assinando um termo de consentimento informado em duas vias.

\section{Controle dos parâmetros dietéticos}

Todos os voluntários preencheram recordatório alimentar de três dias, e foram orientados a não modificarem seus hábitos alimentares durante o protocolo do estudo. Para os dias de teste, foram orientados a realizar uma refeição padrão duas horas antes da sessão de treino de força, composta por $60 \%$ de carboidratos, $15 \%$ de proteínas e $25 \%$ de lipídios, calculada por nutricionista e fornecida previamente aos participantes.

\section{Avaliação da composição corporal e força}

Inicialmente, realizou-se avaliação da composição corporal a partir da aferição da massa corporal, estatura e mensuração das dobras cutâneas (peitoral, abdominal e coxa) para o cálculo do percentual de gordura, conforme o protocolo de Jackson e Pollock ${ }^{(15)}$. Todas as medidas foram realizadas por um mesmo avaliador, que pinçou as dobras de forma intercalada por três vezes e considerou a mediana das medidas para o cálculo. Após este procedimento, os voluntários realizaram aquecimento e foi aplicado o teste de 1$\mathrm{RM}$ estimado. Devido à experiência prévia de todos os voluntários com este teste, não foram realizadas sessões de familiarização. O teste se caracterizou por três tentativas, separadas entre si por 3 minutos de intervalo, em intensidades crescentes até se atingir
10 ou menos repetições com caráter máximo de esforço. A partir desse momento, utilizou-se a tabela de correção de Lombardi(16) para determinar o valor máximo para uma repetição. Foram executadas repetições nos seguintes exercícios: supino livre, puxada pela frente, meio-desenvolvimento, rosca direta, rosca testa, leg press $45^{\circ}$ e agachamento no multi-força.

\section{Suplementação}

A suplementação constituiu-se das seguintes bebidas: bebida carboidrato composta por $1 \mathrm{~g}$ de maltodextrina por quilograma de massa corporal da marca Carb Up ${ }^{\circledR}$ (Probiótica), reconstituída em $250 \mathrm{~mL}$ de água; ou bebida placebo composta de suco com sabor e sem adição de açúcar da marca Clight $^{\circledR}$ (composição: Og de $\mathrm{CHO}$ ) na mesma quantidade da bebida maltodextrina. A ordem de consumo das bebidas foi estipulada de forma randomizada e cega, através de um sorteio prévio, e elas foram oferecidas 15 minutos antes do início da sessão de treino.

\section{Procedimento experimental}

Os voluntários compareceram ao local da avaliação duas horas após a última refeição. A mensuração da glicemia capilar (AccuChek, Roche) foi realizada no lóbulo da orelha, em quatro momentos: antes do consumo da bebida (placebo ou carboidrato), $15 \mathrm{mi}-$ nutos após a ingestão da bebida, metade da sessão e final da sessão. A freqüência cardíaca foi monitorada com cardiotacômetro (POLAR S810). A sessão de treino de força consistiu de três séries de repetições máximas (RM), separadas por um minuto de intervalo passivo, nos exercícios supino livre, puxada pela frente, meio-desenvolvimento, rosca direta, rosca testa, leg press $45^{\circ} \mathrm{e}$ agachamento, numa intensidade de $70 \%$ dos valores de 1-RM. A concentração sérica de lactato foi mensurada 2 minutos após o término da última série da sessão (Accusport, Roche)(17).

Para a avaliação do desempenho, foram utilizados os parâmetros de freqüência cardíaca (FC), tonelagem da sessão de treino e concentração de lactato. Para o cálculo da tonelagem total, multiplicou-se o número de repetições executadas pelo número de séries e pela carga levantada ao longo da sessão de treino.

\section{Estatística}

Os dados foram estruturados e analisados utilizando o pacote estatístico SPSS versão 10.0 para Windows. Os testes de Shapiro-Wilk e Levene foram utilizados para verificar os pressupostos de normalidade e homogeneidade das variâncias, respectivamente.

Para os resultados de glicemia utilizou-se ANOVA Two-Way para medidas repetidas e os resultados de desempenho foram avaliados com teste $t$ para amostras independentes.

Os resultados foram expressos em média \pm desvio padrão e o nível de significância aceito foram de $p<0,05$.

\section{RESULTADOS}

Os dados referentes à caracterização da amostra são descritos na tabela 1, onde verificou-se que a população estudada apresenta índice de massa corporal acima do preconizado para a população saudável, mas justifica-se pelo treinamento de força que realizam mantendo um percentual de gordura adequado.

TABELA 1

Caracterização da amostra

\begin{tabular}{lr}
\hline \multicolumn{1}{c}{ Variáveis } & Média $\pm \mathbf{d p}$ \\
Peso $(\mathrm{Kg})$ & $79,8 \pm 9,2$ \\
Estatura $(\mathrm{cm})$ & $175,3 \pm 6,2$ \\
IMC $\left(\mathrm{kg} / \mathrm{m}^{2}\right)$ & $26,0 \pm 3,0$ \\
Percentual gordura (\%) & $12,9 \pm 4,2$ \\
\hline
\end{tabular}

Valores expressos em média \pm dp dos oito participantes do estudo. 
A figura 1 ilustra o comportamento da glicemia durante o período avaliado em ambos os grupos. Após 15 minutos da ingestão da bebida carboidrato, o grupo apresentou aumento significativo de sua glicemia $(98,25 \pm 17,77 \mathrm{mg} / \mathrm{dL}$ para $133,12 \pm 22,76 \mathrm{mg} / \mathrm{dL}, \mathrm{p}$ $=0,015)$, resposta que não foi encontrada no grupo que ingeriu a bebida placebo $(98,25 \pm 13,69 \mathrm{mg} / \mathrm{dL}$ para $94,38 \pm 12,21 \mathrm{mg} / \mathrm{dL}, \mathrm{p}$ $=1,000)$. Durante o exercício, não houve alteração estatisticamente significativa da glicemia nos indivíduos em ambos os grupos.

\section{GLICEMIA}

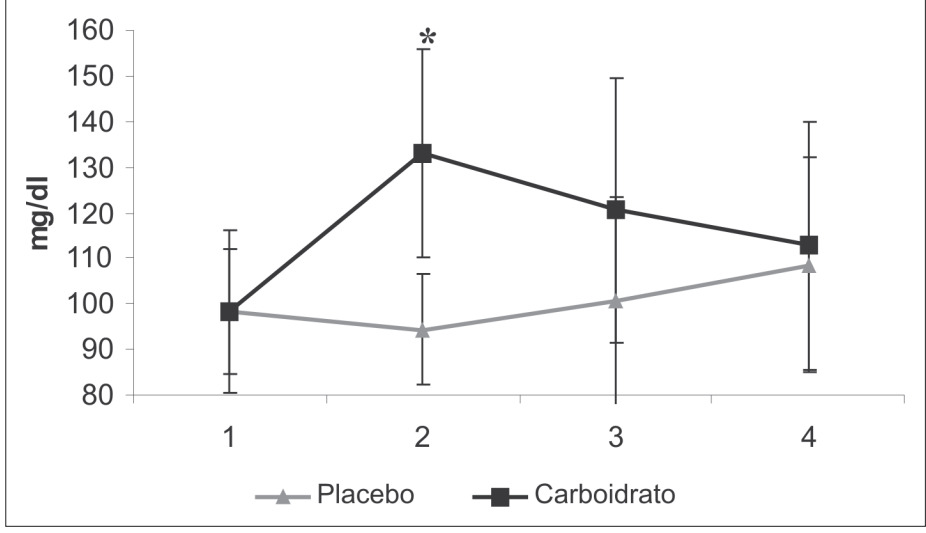

Figura 1 - Glicemia dos grupos placebo e carboidrato nos momentos antes da bebida (1); 15' após a bebida (2); metade do treino (3) e imediatamente após o treino (4). ${ }^{*} p<0,05$ em relação ao momento 1 no mesmo grupo.

A tabela 2 apresenta os resultados do desempenho, avaliados através da FC média durante a sessão de treino, bem como a tonelagem executada durante a sessão e concentração de lactato ao final da sessão. Não foram verificadas diferenças significativas nestas variáveis quando comparados os dois grupos, demonstrando que a bebida não interferiu nestas variáveis.

\section{TABELA 2}

Resultados relacionados ao desempenho

\begin{tabular}{lcc}
\hline & Placebo & Carboidrato \\
& $12876,3 \pm 2024,8$ & $12944,3 \pm 2547,9$ \\
Tonelagem $(\mathrm{kg})$ & $135 \pm 13$ & $136 \pm 15$ \\
FC média $(\mathrm{bpm})$ & $6,7 \pm 2,5$ & $7,3 \pm 2,6$ \\
Concentração lactato $(\mathrm{mmol} / \mathrm{L})$ & \\
\hline
\end{tabular}

Valores da freqüência cardíaca $(\mathrm{FC})$ média durante a sessão de treino de força, concentração de lactato ao final do treino de força e tonelagem para os grupos placebo e carboidrato. Valores expressos em média \pm desvio padrão.

\section{DISCUSSÃO}

O principal achado deste estudo foi que a suplementação prévia com $1 \mathrm{~g} / \mathrm{kg}$ corporal de carboidrato aumentou a glicemia após 15 minutos do consumo, mas não alterou a glicemia e o desempenho físico de indivíduos treinados durante uma sessão subseqüente de treino de força. Nossos resultados não sustentam a hipótese de que a ingestão prévia de carboidrato poderia influenciar o conteúdo muscular de glicogênio, e assim desempenhar seu efeito potencial no retardo da fadiga durante o treino de força. Além disto, não ocorreram alterações significativas entre as duas sessões de exercícios para outras variáveis como concentração final de lactato e freqüência cardíaca durante o treino, as quais poderiam indicar diferenças de intensidade.

Analisando-se o comportamento da glicemia, para o grupo placebo, nossos resultados corroboram com os de Haff et al. ${ }^{(13)}$ e os de Keul et al. ${ }^{(18)}$, nos quais o treinamento de força, não provocou alterações significativas na glicemia durante a sessão de treino.
Os resultados da glicemia, após a ingestão de carboidratos foram semelhantes aos de Haff et al.(4,13) e aos de Conley et al..(19), apresentando uma elevação significativa da glicemia. No entanto, nosso estudo apresentou aumento da glicemia apenas entre os momentos 1 (antes da bebida) e 2 (15 minutos após a bebida), tendo a glicemia sido regularizada durante a sessão de treino. O mesmo comportamento da glicemia foi observado em um estudo prévio realizado pelo nosso grupo com suplementação de carboidratos e exercício de resistência(20).

Ao contrário do presente trabalho, os estudos de Haff et al. $(4,13)$, Conley et al..(19) e Bird et al.(21-22) apresentaram a glicemia aumentada durante todo o período da sessão de treino. Isto provavelmente se deve ao fato de que nosso estudo fez uso da ingestão de carboidratos apenas antes das sessões de treino, enquanto que os demais estudos citados forneceram carboidratos antes e durante as sessões.

Em relação ao desempenho, nossos resultados corroboram com os de Haff et al.(13), que não encontraram diferenças significativas com a suplementação de carboidratos em relação ao placebo. No estudo de Haff, os sujeitos realizaram uma sessão de três séries de 10 repetições de extensão do joelho, isocineticamente, a $120^{\circ} . \mathrm{s}^{-1}$, que foi usada como marcador de performance, antes e depois de uma sessão de treino com pesos livres. Nossos resultados também vão ao encontro dos resultados de Conley et al.(19), onde os sujeitos realizaram uma sessão de treino que consistia em séries de 10 repetições a $65 \%$ de 1-RM até a exaustão. Os indivíduos consumiram uma bebida a base de carboidratos $15 \mathrm{mi}$ nutos antes do início do treino e bebiam novamente a cada série completada com sucesso. Em um outro momento, os mesmos sujeitos executaram o mesmo protocolo, porém ingerindo placebo. Não houve diferenças significativas no número de repetições, séries e tonelagem total entre as soluções. Um outro estudo que apresentou resultados semelhantes aos nossos, no quesito desempenho, foi o de Vincent et al. ${ }^{(14)}$, no qual os sujeitos faziam uma ingestão de carboidratos imediatamente antes do início de uma sessão de 8 exercícios de força com pesos livres. Neste estudo também foi usado o exercício isocinético de perna, após a sessão de treino, como método de avaliação do desempenho, e nenhuma alteração significativa foi verificada em função da ingestão de carboidratos. Utter et al. ${ }^{(23)}$ também não verificaram melhoras no desempenho verificados através da Escala de Percepção de Esforço de Borg após consumo de carboidratos.

Alguns estudos encontraram resultados diferentes dos apresentados no presente trabalho. Lambert et al.(24) demonstraram que a ingestão de carboidratos, antes e durante a realização da sessão de treino consistida de séries de 10 repetições a $80 \%$ de 1-RM de extensão do joelho até a exaustão, aumentava significativamente a capacidade de realização de séries e de repetições em relação ao placebo. Haff et al.(4), em outro de seus estudos, encontraram melhoras na performance com a ingestão prévia de carboidratos em uma sessão de 16 séries de 10 repetições do exercício extensão do joelho, realizado isocineticamente a $120^{\circ} . \mathrm{s}^{-1}$, comparado com a ingestão de placebo.

Não se pode definir as causas para essas diferenças de resultados encontradas nos diversos estudos. No entanto, há algumas possibilidades que podem ser discutidas. Uma delas é o tempo de duração das sessões de treino. Os estudos de Haff et al.(4) e o de Lambert et al.(24), que apresentaram diferenças significativas no desempenho, devido à ingestão de carboidratos, tiveram um tempo de duração das sessões de treino de 57 e 56 minutos respectivamente, em contrapartida os estudos de Conley et al. ${ }^{(19)}$ e o de Haff et al.(13), que não apresentaram diferenças significativas na performance, tiveram um tempo de duração das sessões de treino de 35 e 39 minutos, respectivamente. Em nosso estudo, a sessão de treino teve duração média de 45 minutos. Sendo assim, é possível que a duração do treino tenha influenciado a efetividade da suplementação de carboidratos como recurso ergogê- 
nico. Anantaraman et al.(25) afirmam em seu estudo que sessões de treino de força com menos de 40 minutos utilizam o glicogênio muscular como principal substrato energético, e que à medida que o exercício segue (após esses 40 minutos) diminui-se a utilização do glicogênio muscular e aumenta-se a utilização da glicose sanguínea exógena. O autor sugere que o volume de trabalho executado seja algo significativo para a efetividade da suplementação de carboidrato como fator otimizante de desempenho. Sugere-se também, que é possível que atividades com altos volumes, maiores que 40 minutos estimulem um estresse maior no sistema glicogenólico.

Outra diferença na metodologia dos estudos diz respeito à quantidade e momentos da ingestão de carboidratos. Alguns, como o nosso, utilizaram a ingestão de carboidratos apenas antes da sessão de treino, outros utilizaram antes e durante a sessão, o que resultou em consumos bem maiores. Entretanto, também não se pode chegar a um consenso quanto a isso, visto que tanto Lambert et al.(24) quanto Conley et al.(19) utilizaram ingestão de carboidratos antes e durante a sessão de treino, e apesar de terem utilizados protocolos de treino parecidos, obtiveram resultados diferentes quanto ao desempenho. Coyle et al.(26) e Bergstrom et al. ${ }^{(10)}$ sugerem que esta ingestão durante a sessão de treino, em atividades com altos volumes, pode poupar o glicogênio muscular ou fazer da glicose sanguínea o principal substrato energético à medida que o glicogênio muscular é depletado.

No presente estudo não verificou-se diferença significativa da FC durante as duas sessões de exercício de força, efeito semeIhante ao que é encontrado com exercício aeróbico realizado após de ingestão de carboidrato ou placebo(27-29). Comparando o lactato sanguíneo, que pode ser usado como importante marcador de intensidade de exercício(30), com estudos com exercícios de endurance, nossos resultados corroboram com os de Gleeson et al.(31), que não encontraram diferenças significativas na concentração final de lactato para as diferentes soluções. Assim como no estudo de Sapata et al.(20) no qual a suplementação com carboidrato previamente ao exercício não alterou as concentrações finais de lactato. A não alteração nas concentrações de lactato sanguíneo neste estudo sugere que as duas sessões de treino tiveram intensidades muito semelhantes.

\section{REFERÊNCIAS}

1. Febbraio MA, Stewart KL. CHO feeding before prolonged exercise: effect of glycemic index on muscle glycogenolysis and exercise performance. J Appl Physiol. 1996;81(3):1115-20.

2. Kirwan JP, O'Gorman D, Evans WJ. A moderate glycemic meal before endurance exercise can enhance performance. J Appl Physiol. 1998;84(1):53-9.

3. Febbraio MA, Keenan J, Angus DJ, Campbell SE, Garnham AP. Preexercise carbohydrate ingestion, glucose kinetics, and muscle glycogen use: effect of the glycemic index. J Appl Physiol. 2000;89(5):1845-51.

4. Haff GG, Schroeder CA, Koch AJ, Kuphal KE, Comeau MJ, Potteiger JA. The effects of supplemental carbohydrate ingestion on intermittent isokinetic leg exercise. J Sports Med Phys Fitness. 2001;41(2):216-22.

5. Rowlands DS, Hopkins WG. Effect of high-fat, high-carbohydrate, and high-protein meals on metabolism and performance during endurance cycling. Int J Sport Nutr Exerc Metab. 2002;12(3):318-35.

6. Wright DA, Sherman WM, Dernbach AR. Carbohydrate feedings before, during, or in combination improve cycling endurance performance. J Appl Physiol. 1991; $71(3): 1082-8$.

7. Haff GG, Lehmkuhl MJ, McCoy LB, Stone MH. Carbohydrate supplementation and resistance training. J Strength Cond Res. 2003;17(1):187-96.

8. Robergs RA, Pearson DR, Costill DL, Fink WJ, Pascoe DD, Benedict MA, et al. Muscle glycogenolysis during differing intensities of weight-resistance exercise. J Appl Physiol. 1991;70(4):1700-6.

9. Steensberg A, van Hall G, Keller C, Osada T, Schjerling P, Pedersen BK, et al. Muscle glycogen content and glucose uptake during exercise in humans: influence of prior exercise and dietary manipulation. J Physiol. 2002;541(Pt 1):273-81.

10. Bergstrom J, Hultman E. A study of the glycogen metabolism during exercise in man. Scand J Clin Lab Invest. 1967;19(3):218-28.
Por ser um estudo experimental, este também apresenta limitações. O tamanho da amostra dificulta a extrapolação dos resultados, assim como dificulta a análise estatística dos resultados apresentados. Outra limitação seria o uso da glicemia capilar para avaliação da glicemia sanguínea. Entretanto, com a preocupação de avaliar a precisão e exatidão dos resultados oferecidos pelos glicosímetros, estudos foram realizados visando comparar os resultados do equipamento com os resultados obtidos por ensaios laboratoriais ${ }^{(32-33)}$. Os resultados revelaram que os resultados obtidos pelos glicosímetros foram exatos, com comparação dos valores de glicemia capilar medida pelo glicosímetro com glicemia venosa e capilar medida no laboratório resultou em coeficientes de correlação de 0,9819 e 0,9842, respectivamente ${ }^{(32)}$. Porém, os autores alertam para o fato de que os pacientes devem receber treinamento para o correto manuseio do equipamento, o que é corroborado pela literatura(34).

Em conclusão, este estudo demonstrou que a ingestão de uma solução de carboidrato $(1 \mathrm{~g} / \mathrm{kg}$ peso), 15 minutos antes de uma sessão de treino de força, consistindo de 3 séries de 7 exercícios a 70 \% de 1-RM até a exaustão, não foi capaz de alterar o desempenho. A glicemia, apesar de sofrer um aumento significativo, após a ingestão do carboidrato, retornou a valores próximos aos iniciais e assim se mantém até o fim da sessão, não apresentando nenhuma queda abrupta, o que caracterizaria o fenômeno da hipoglicemia de rebote. O estudo demonstrou também, que a concentração final de lactato e a freqüência cardíaca média não são modificadas significativamente pela solução de carboidrato e apresentaram valores estatisticamente semelhantes aos da solução placebo. São necessários outros estudos para que se possa entender melhor a suplementação prévia de carboidratos e ao longo de uma sessão de treinamento de força, bem como os mecanismos de interação desta suplementação com as variáveis pertinentes ao treinamento de força, tais como, freqüência cardíaca, concentração de lactato, desempenho, comportamento da glicemia, entre outras.

Todos os autores declararam não haver qualquer potencial conflito de interesses referente a este artigo.
11. Yaspelkis BB 3rd, Singh MK, Trevino B, Krisan AD, Collins DE. Resistance training increases glucose uptake and transport in rat skeletal muscle. Acta Physiol Scand. 2002;175(4):315-23.

12. Burke LM, Claassen A, Hawley JA, Noakes TD. Carbohydrate intake during prolonged cycling minimizes effect of glycemic index of preexercise meal. J Appl Physiol. 1998;85(6):2220-6.

13. Haff GG, Koch AJ, Potteiger JA, Kuphal KE, Magee LM, Green SB, et al. Carbohydrate supplementation attenuates muscle glycogen loss during acute bouts of resistance exercise. Int J Sport Nutr Exerc Metab. 2000;10(3):326-39.

14. Vincent KR CP, Freedson PS, Decheke M. Effects of pre-exercise liquid, high carbohydrate feeding on resistance exercise performance. Med Sci Sports Exerc. 1993;25:S194

15. Jackson AS, Pollock ML. Generalized equations for predicting body density of men. Br J Nutr. 1978;40(3):497-504.

16. Lombardi V. Beginning weight training. Debuque: Ed. Brown; 1989

17. Bishop D. Evaluation of the Accusport lactate analyser. Int J Sports Med. 2001; 22(7):525-30.

18. Keul J, Haralambie G, Bruder M, Gottstein HJ. The effect of weight lifting exercise on heart rate and metabolism in experienced weight lifters. Med Sci Sports. 1978;10(1):13-5.

19. Conley M, Stone M, Marsit, JL, O'Bryant, HS, Nieman, DC, Johnson, JL, et al. Effects of carbohydrate ingestion on resistance exercise. J Strength Cond Res. 1995;9:20

20. Sapata KB FA, Oliveira AR. Efeitos do consumo prévio de carboidratos sobre a resposta glicêmica e desempenho. Rev Bras Med Esporte. 2006;12(4):189-94.

21. Bird SP, Tarpenning KM, Marino FE. Effects of liquid carbohydrate/essential amino acid ingestion on acute hormonal response during a single bout of resistance exercise in untrained men. Nutrition. 2006; 22(4):367-75. 
22. Bird SP, Tarpenning KM, Marino FE. Independent and combined effects of liquid carbohydrate/essential amino acid ingestion on hormonal and muscular adaptations following resistance training in untrained men. Eur J Appl Physiol. 2006; 97(2):225-38.

23. Utter AC, Kang J, Nieman DC, Brown VA, Dumke CL, McAnulty SR, et al. Carbohydrate supplementation and perceived exertion during resistance exercise. Strength Cond Res. 2005;19(4):939-43.

24. Lambert C, Flynn M, Boone J, Michaud T, Rodriguez-Zayas J. Effects of carbohydrate feeding on multiple-bout resistance exercise. J Appl Sport Sci Res. 1991; 5:192-7.

25. Anantaraman R, Carmines AA, Gaesser GA, Weltman A. Effects of carbohydrate supplementation on performance during 1 hour of high-intensity exercise. Int $\mathrm{J}$ Sports Med. 1995;16(7):461-5

26. Coyle EF, Coggan AR, Hemmert MK, Ivy JL. Muscle glycogen utilization during prolonged strenuous exercise when fed carbohydrate. J Appl Physiol. 1986;61(1): 165-72.

27. Fielding RA, Costill DL, Fink WJ, King DS, Kovaleski JE, Kirwan JP. Effects of preexercise carbohydrate feedings on muscle glycogen use during exercise in welltrained runners. Eur J Appl Physiol Occup Physiol. 1987;56(2):225-9.
28. Kirwan JP, O'Gorman DJ, Cyr-Campbell D, Campbell WW, Yarasheski KE, Evans WJ. Effects of a moderate glycemic meal on exercise duration and substrate utilization. Med Sci Sports Exerc. 2001;33(9):1517-23.

29. Sparks MJ, Selig SS, Febbraio MA. Pre-exercise carbohydrate ingestion: effect of the glycemic index on endurance exercise performance. Med Sci Sports Exerc. 1998;30(6):844-9

30. Myers J, Ashley E. Dangerous curves. A perspective on exercise, lactate, and the anaerobic threshold. Chest. 1997;111(3):787-95.

31. Gleeson M, Maughan RJ, Greenhaff PL. Comparison of the effects of pre-exercise feeding of glucose, glycerol and placebo on endurance and fuel homeostasis in man. Eur J Appl Physiol Occup Physiol. 1986;55(6):645-53.

32. Mira GS, Candido LM, Yale JF. Performance of glucometer used for self-monitoring blood glycaemia in type 1 diabetic patients. Arq Bras Endocrinol Metabol. 2006;50(3):541-9.

33. Velazquez Medina D, Climent C. Comparison of outpatient point of care glucose testing vs venous glucose in the clinical laboratory. P R Health Sci J. 2003;22(4): 385-9.

34. Kendall DM, Kaplan RA, Paulson CF, Parkes JL, Tideman AM. Accuracy and utility of a 10-test disk blood glucose meter. Diabetes Res Clin Pract. 2005;67(1): 29-35. 